\title{
28 Research Square \\ Sleep Medication Use and Risk of Fractures in Breast Cancer Survivors
}

Reina Haque ( $\square$ reina.haque@kp.org)

Kaiser Permanente Southern California https://orcid.org/0000-0001-8563-3330

Rowan T. Chlebowski

The Lundquist Institute

LieHong Chen

Kaiser Permanente Southern California

\section{Research Article}

Keywords: breast cancer, sleep medication, fractures, comorbidity

Posted Date: June 21st, 2021

DOI: https://doi.org/10.21203/rs.3.rs-614637/v1

License: (1) This work is licensed under a Creative Commons Attribution 4.0 International License.

Read Full License 


\section{Abstract}

Purpose: Sleep problems are more common in breast cancer survivors than those without a cancer history. Our goal was to examine the risk of fractures among breast cancers survivors who used prescription sleep aids.

Methods: We conducted a retrospective cohort study of 21,346 adult women diagnosed with stage 0 -III breast cancerbetween 2009-2016and followed them through 2017. We examined person-year rates of fracturesby sleep medication use and calculated adjusted hazard ratios (HR) and 95\% confidence intervals $(\mathrm{Cl})$ with Cox proportional hazards models using time-dependent variables for sleep medications and covariate medications (antidepressants, anti-anxiety medications, and bisphosphonates) adjusted for demographics, comorbidities, and tumor characteristics and cancer treatments.

Results: The sleep medication use was common (40\%) in breast cancer survivors and was associated with a $33 \%$ increased risk of fractures(adjusted HR=1.33, 95\% Cl: 1.20-1.49). Bisphosphonateuse did not mitigate this risk. Further, in a sensitivity analysis based on new use of sleep medication, the fracture risk was even stronger (adjusted HR=1.44, 95\% Cl: 1.26-1.64).

Conclusion:Given the high use of sleep medicationsand the high risk of fractures in breast cancer survivors, this study suggests that non-pharmacologic management of sleep problems might be considered as alternative therapy.

\section{Introduction}

For many of the 3.8 million femalebreast cancer survivors living in the US in 2019 ,improved survival is complicated by long-term psychosocial effects including sleep problems[1].Theprevalence of insomnia symptoms is nearly $40 \%$ in cancer survivors versus $10-15 \%$ in the general U.S. population [2]. Studies suggest that sleep problems arise due to the side effects of cancer treatments or due to the disease itself [3]and can persist years after primary cancer therapy completion [4]. Breast cancer survivors are more likely to have physical symptoms and psychological distress than patients with other types of cancers possibly due the treatment side effects [5-6]. For example, hot flashes caused by tamoxifen can lead to or exacerbatesleep problems in breast cancer survivors [7]. However, limited information exists about use of sleep aids in breast cancer survivors and consequent health outcomes.

Prescription sleep aids include benzodiazepines, non-benzodiazepines acting on benzodiazepine receptors, and antidepressants. Furthermore, new research suggests the risk of hip fractures rises soon after an older person is prescribed a sleeping pill [8].Given this background, breast cancer survivors may have an even greater risk of fractures due to the bone loss induced by adjuvant hormonal therapy (aromatase inhibitors), toxicity of the chemotherapies themselves, and/or greater use of sleep aids to cope with sleep problems. 
Previous studies that examined the adverse health effects of sleep medications in cancer survivors were limited because they included data from a single academic hospital, were based on patient with various types of cancer, or did not address confounding factors such as comorbidities or use of antidepressants [9-13].In sum, sparse population-13based data exist about theseserious sequelae of prescription sleep medication use in breast cancer survivors. Thus, our objective was to examine fracture risk in breast cancer survivors who used sleep medications, while accounting for antidepressant use, psychosocial status, osteoporosis, other comorbidities, tumor characteristics,adjuvant cancer, and bisphosphonates (bone enhancing drugs).This knowledge can inform interdisciplinary clinical management of tens of thousands breast cancer survivors experiencing sleep problems as result of their disease, side-effects of cancer treatments, or distress.

\section{Methods}

\section{Data sources and setting}

This study was conducted at Kaiser Permanente Southern California (KPSC), a large managed care system that comprises nearly 15 hospitals and over 200 medical clinics that servewith 4.7 million members. Patients receive virtually all their medical care and prescription drugs within this integrated healthcare delivery system, and information on any outside procedures and diagnoses are available through claims databases. The health plan's National Cancer Institute-Surveillance, Epidemiology, and End Results (SEER)-affiliated tumor registry was used to identify subjects with breast cancer. The KPSC Institutional Review Board reviewed and approved this study.

\section{Subjects and design}

We assembled a cohort of women diagnosed with first primary breast cancer in 2009-2016 followed through December 2017. Eligible women included adults (>18 years at diagnosis), with American Joint Commission on Cancer TNM Stage 0 -III breast cancer, and with at least one year of continuous membership prior to their cancer diagnosis $(n=21,513)$. We excluded 167 women with a history of fractures in the prior 3 months before breast cancer diagnosis to reduce confounding, leaving 21,346 for analysis.

\section{Incident fractures outcomes}

We identified first incident fracturesthat occurred after breast cancer diagnosis, from the health plan's electronic health records (EHR). Fractures of the forearm, femur, lower leg, wrist and hand, vertebrae, and pelvis were identified using ICD9 (International Classification of Diseases, Ninth Revision) codes and ICD10 codes(see supplement table for details). The event that occurred first served as the outcome.

\section{Sleep medications}

The pharmacy dispensing database was used to capture use of all sleep aids (name, date of initiation, days supplied) in the health plan's formulary: lorazepam, trazodone, doxepin, flurazepam, temazepam, 
triazolam, eszopiclone, zaleplon, zolpidem, and suvorexant. We also considered the effect of "new use" of the sleep medication on risk of fracture. "New use" was defined as the drug dispensing that occurred without drug possession in the preceding 3 monthsbefore breast cancer diagnosis.

\section{Covariates}

A comprehensive set of covariates was captured from the EMR. These included race/ethnicity and tumor factors (age and stage at breast cancer diagnosis, adjuvant cancer therapy, diagnosis year, and tumor characteristics). We also captured prior comorbidities from one-year before breast cancer diagnosis, and current comorbiditiespost breast cancer diagnosis through the end of each woman's follow-up. Current comorbidities ascertained included osteoporosis,hypertension, depression, dementia, anxiety, and sleep problems. We extracted data on covariate medications such as antidepressant, anti-anxiety, and bisphosphonate use during the follow-up period. Bisphosphonates (alendronate, which was the most commonly useddrug in $93 \%$ of patients)was prescribed to combat the bone loss typically associated with adjuvant aromatase inhibitor therapy.

\section{Statistical analyses}

Follow-up commenced on the breast cancer diagnosis date and censored on the date of the earliest study endpoint: first fracture diagnosis date, death, termination of health plan membership, or study's end (December 31, 2017). The definition of continuous health plan enrollment allowed for gaps of up to 3 months in enrollment during study period, as these were likely administrative gaps. In descriptive analyses, we compared the distribution of all variables (demographics, tumor characteristics, comorbidities, and covariate drugs) by fracture status and sleep medication use. The chi-square or Fisher exact tests were used to compare categorical variables, and the Kruskall-Wallis tests was used for continuous variables.Because women were followed different lengths of time, we computed the personyear rates of fractures. Odds ratios and $95 \%$ confidence intervals $(\mathrm{Cl})$ were used to compute the association of mental health conditions with use of sleep medications and correlations between sleep medications and psychiatric medications.

Crude and adjusted hazard ratios (HR) and 95\% Cls were estimated for fracture riskby Cox proportional hazards models using time-dependent variables for sleep aids and the other covariate medications (antidepressants, anti-anxiety medications, bisphosphonates)used during the follow-up period. We also adjusted for adjuvant endocrine therapy (tamoxifen and aromatase inhibitors treated as time-dependent) in the models. All covariates selected for adjustment in the model were based on clinical importance and descriptive statistics. The proportional hazards assumption was tested via graphic plots and Schoenfeld residuals; no violations were found.

We also conducted additional analyses to assess the robustness of the multivariable results of the association between sleep medication use and fracture risk. First, we evaluated two models stratified by bisphosphonate use status using the full cohort $(n=21,346)$. Second, we repeated analyses on the subset of women who were "new users" of sleep medications ( $n=16,486)$. Except for body mass index $(B M I,<5 \%$ 
was missing), missing data was rare for the study variables. Unknown BMI was entered as a category in the multivariable models (as done for race/ethnicity which had 1\% missing in the other/mixed/unknown category). All analyses were performed using SAS Version 9.4 (SAS Institute, Cary NC).

\section{Results}

In the cohort 21,346breast cancer survivors, we observed 2,038 incident fractures deaths during the 85,425 person-years of follow-up (median of 3.6years [interquartile range,IQR: 2.0-5.9]). The median age at breast cancer diagnosis was 62.0 years (IQR: $53.0-70.0$ ). The cohort was diverse with $51.9 \%$ nonHispanic White; 20.4\% Hispanic; 13.2\% African American/Black; 13.5\% Asian/Pacific Islanders; and 1.1\% of mixed/unknown race/ethnicity (Table 1).Those who suffered fractures were more likely to be aged $>65 y e a r s$, non-Hispanic White, and have comorbid depression, anxiety, dementia or sleep problems compared to those who did not have fractures ( $\mathrm{P}<0.001$ for all variables). Those who experienced fractures were also more likely to have used antidepressants (32.4\%) than those who did not have fractures $(23.2 \%)$.

In Table 2, women who had fractures were more likely to have invasive breast cancer (Stages I-III, 83.1\%) than those who did not have fractures $(78.8 \%)$. In terms of cancer therapy, those who underwent adjuvant chemotherapy were less likely to have fractures $(P<0.001)$, but those who usedadjuvant hormonal therapy $(P=0.004)$ were more likely to experiencefractures. We did not find a statistically significant difference by adjuvant radiation therapy $(\mathrm{P}=0.09)$.

\section{Sleep Aid Medication Use}

Roughly $40 \%$ of the cohort used prescription sleep aid medications $(38.5 \%, 8,225 / 21,346)$ for a median of 60.0 days (IQR: 15.0-268.0) (Table 3). The most commonly used sleep aid medication was lorazepam (used by $n=5819$ women), followed by trazodone $(n=2296)$, and temazepam $(n=1615)$. As shown in Table 3 , women used multiple types of sleep medications. Overall, the median cumulative duration of sleep medication use was 60 days (interquartile range, IQR: 15-268 days). Sleep aid medication use was strongly correlated with anti-anxiety medication use ( $\mathrm{OR}=3.65,95 \% \mathrm{Cl}$ : 3.36-3.97); antidepressant use $(\mathrm{OR}=3.97 \% \mathrm{Cl}$ : $3.69-4.28)$; and use of both of these medications (OR=11.07, 95\% Cl: 0.08-12.17).Breast cancer survivors who used sleep aid medications were more likely to have depression ( $40.5 \%$ vs. $17.2 \%$, $\mathrm{OR}=3.27,95 \% \mathrm{Cl}: 3.07-3.49)$ and anxiety (57.3\% vs. $21.4 \%$; $\mathrm{OR}=4.94,95 \% \mathrm{Cl}: 4.65-5.25)$ than non-users (Table 4). The most common antidepressants used were selective serotonin reuptake inhibitors (SSRI), followed by tricyclic antidepressants (TCA), and serotonin-norepinephrine reuptake inhibitors (SNRI); most women used multiple classes of antidepressants during follow-up (Table 4).

\section{Fracture Risk}

Sleep aid medication use was associated with a greater fracture rate in breast cancer survivors (28.62/1,000 person-years) than for non-use (20.52/1,000 person-years) (Table 5). Correspondingly, in the multivariable Cox proportional hazards model that handled medication use as time-varying, sleep aid 
medication use was associated with a $33 \%$ increased fracture risk (adjusted $\mathrm{HR}=1.33$ [95\% Cl: 1.19-1.48], $P<0.001)$ after adjusting for race/ethnicity, age, body mass index, cancer stage, adjuvant cancer therapy, year of diagnosis,osteoporosis, depression, anxiety, dementia, Charlson Comorbidity Index, and use of antidepressants and anti-anxiety medications. This association was similar after we stratified by bisphosphonate use (adjusted HR for sleep medication and fractures $=1.36$ [95\% Cl: 1.14-1.62]for bisphosphonate use, and $\mathrm{HR}=1.32[95 \% \mathrm{Cl}: 1.14-1.51]$ for bisphosphonate non-use). In the additional sensitivity analyses based on the subset of "new use" of sleep aid medications, anti-anxiety and antidepressants $(n=16,486)$, the association was even stronger with an adjusted $\mathrm{HR}=1.44$ [95\% Cl: 1.261.64](Table 5). The strong association between sleep aid medications and fracture risk persisted for bisphosphonate use(adjusted $\mathrm{HR}=1.51$ [95\% Cl: 1.22-1.87]) and bisphosphonate non-use (adjusted $\mathrm{HR}=1.39$ [95\% Cl: 1.17-1.64]).

\section{Discussion}

Among 21,346 breast cancer patients without prior fractures, prescription sleep aid medication use was associated with a $33 \%$ increased risk of fractures during the 85,425 person-years of follow-up (median of 4.0 years [interquartile range: 2.2-6.1 years]). Additionally, bisphosphonate use did not mitigate fracture risk. Furthermore, in a sensitivity analysis based on breast cancer survivors with new use of sleep aid medications, the fracture risk was $44 \%$ greater. To our knowledge, our study is the first to integrate comprehensive set of covariates in examining the fractures risk related to sleep aid medication usein a large community-based sample of breast cancer survivors. Our results suggest that prescription sleep aids increase the fracture risk in this vulnerable population.

Reasons for our findings relate to the hypnotic properties of the sleep medications that linger through the day exacerbating the risk of falls. In addition,breast cancer survivors taking aromatase inhibitors (Als) might also be vulnerable to this effect given that Al's are associated with acceleration of bone loss and an increasedrisk of osteoporotic fractures. Due to the adverse bone effects associated with Als, patients are given bisphosphonates to combat this, but our study did not find a marked protection from bisphosphonates. Similarly, other epidemiologic studies have not found a protective effect of bisphosphonates in reducing fractures risk [14-16].

Our study has several strengths. The large sample size and comprehensive healthcare coverage enabled us to examine the fracture risk without concerns about variable healthcare insurance coverage that is known to affect cancer outcomes. Importantly, our analysis was based on filled prescriptions which ensured accuracy for both exposure and covariate drug ascertainment. Further, the study had sufficient long follow-up time to identify fracture risk, with a median of 4 years, ranging up to 9 years. Finally, we applied different analytic strategies to address various sources of bias in observational studies, and we conducted sensitivity analysesto assure the robustness of the conclusion. The HR estimates in our study accounted for demographics, comorbidities, tumor characteristics, cancer treatments, and concurrent medications. In the multivariable Cox proportional hazards model, medications were handled as timedependent thus reducing drug exposure misclassification. Moreover, our sensitivity analyses restricted to 
women who were new users of sleep medications; this further strengthened the finding of the association between sleep medications and fracture risk. Lastly, about half of the breast cancer survivors were from minority groups, which enhances generalizability of our results to the larger California population. Thus, our carefully designed longitudinal analysis better addressed the adverse side-effects of prescription sleep medication in breast cancer survivors in real-world practices.

Certain limitations must be considered. We did not have complete data on BMI, and those with lower BMI might be more likely to have fractures. However, BMI may not be correlated with sleep medication use, thus the effect of this confounder might be minimal. Based on randomized clinical trials data that favored Als over tamoxifen, physicians might have preferentially prescribed Als to older patients, or to those with concerns for [17]. Although we adjusted for adjuvant endocrine therapy (Als and tamoxifen) and examined a comprehensive set of covariates, including time-varying sleep medications, antidepressants, anti-anxiety drugs, residual confounding cannot be precluded in our study.

In summary, we determined that almost $40 \%$ of breast cancer survivors had used sleep aid medications and its use increased the risk of fractures by $33 \%$. This study bridged the gap between studies that examined this question in other vulnerable groups such as older women, but did not specifically include breast cancer survivors. Thus, clinicians must consider the safety of prescribing sleep medications in this vulnerable group. Our findings are also relevant to medical oncologists because they are increasingly providing general care to their patients [19]. Given sleep medications strong association with fractures and that breast cancer survivors taking Als are a susceptible given the Al's adverse bone effects, another implication of this study is thatsleep problems might be better resolved by non-pharmacologic approaches such as cognitive behavioral therapy, increasing daytime physical activity, and limiting caffeine and alcohol.

\section{Conclusion}

Our study demonstrates a large fraction of breast cancer survivors (40\%) used prescription sleep medications, and the most common was lorazepam, a benzodiazepine. Given that new users of sleep medications had a $40 \%$ increased fracture risk, caution is warranted when treating breast cancer survivors for sleep disturbance. Further, bisphosphonates did not mitigate the fracture risk. Nonpharmacologic therapy may be considered to manage the sleep disturbances in breast cancer survivors that arise from distress or due to the side-effects of cancer treatments.

\section{Declarations}

Funding. This study was supported by Kaiser Permanente Southern California, Research \& Evaluation

\section{Conflicts of interest/Competing interests. Not applicable}

Availability of the data. Inquiries for a de-identified analytic dataset can be made to writing with the corresponding author. Data use agreements will be needed between KPSC and the requesting institutes 
as well as KPSC IRB review.

\section{Code availability. Not applicable}

Authors' contributions Funding, manuscript preparation, development of analysis plan (RH); manuscript preparation, data analysis (LC); manuscript preparation, development of analysis plan (RTC)

\section{References}

1. American Cancer Society. Cancer Facts and Figures (2019) Atlanta, GA: American Cancer Society; 2019

2. Palesh OG, Roscoe JA, Mustian KM, Roth T, Savard J, Ancoli-Israel S, Heckler C, Purnell JQ, Janelsins MC, Morrow GR (2010) Prevalence, demographics, and psychological associations of sleep disruption in patients with cancer: University of Rochester Cancer Center-Community Clinical Oncology Program. J Clin Oncol 28(2):292-298. doi:10.1200/JC0.2009.22.5011

3. Bower JE (2008) Behavioral symptoms in breast cancer patients and survivors: Fatigue, insomnia, depression, and cognitive disturbance. J Clin Oncol 26(5):768-777

4. Savard J, Simard S, Blanchet J, Ivers H, Morin CM (2001) Prevalence, clinical characteristics, and risk factors for insomnia in the context of breast cancer. Sleep 24:583-590

5. Li L, Wu C, Gan Y et al (2016) Insomnia and the risk of depression: a meta-analysis of prospective cohort studies. BMC Psychiatry 16:375

6. Savard J, Simard S, Ivers H, Morin CM (2005) Randomized Study on the Efficacy of CognitiveBehavioral Therapy for Insomnia Secondary to Breast Cancer, Part I: Sleep and Psychological Effects. J Clin Oncol 23:6083-6096

7. Haque R, Shi J, Schottinger JE, Ahmed SA, Cheetham TC, Chung J, Avila C, Kleinman K, Laurel A. Habel LA, Fletcher SW, Kwan ML (2016) Tamoxifen and antidepressant drug interaction among a cohort of 16887 breast cancer survivors. JNCl: Journal of the National Cancer Institute 108(3):djv337

8. Berry SD, Lee Y, Cai S, Dore DD (2013) Nonbenzodiazepine sleep medication use and hip fractures in nursing home residents. JAMA Intern Med 173(9):754-761. doi:10.1001/jamainternmed.2013.3795

9. Savard J, Morin CM (2001) Insomnia in the context of cancer: a review of a neglected problem. J Clin Oncol 19:895-908

10. Savard J, Ivers H, Villa J, Caplette-Gingras A, Morin CM (2011) Natural course of insomnia comorbid with cancer: an 18-month longitudinal study. J Clin Oncol 29(26):3580-3586. doi:10.1200/JCO.2010.33.2247

11. Chang WP, Lin CC (2015) Use of opioid analgesics or sleeping medication and survival of cancer patients. Eur J of Oncology Nursing 19(3):199-206. doi:10.1016/j.ejon.2014.11.007

12. Rumble ME, Keefe EJ, Edinger JD, Porter LS, Garst JL. A pilot study investigating the utility of the cognitive-behavioral model of insomnia in early-stage lung cancer patients. $\mathrm{J}$ of Pain and Symptom 
Management. 2005:30;160-169

13. van den Beuken-van Everdignen MH, de Rijke JM, Kessels AG, Schouten HC, van Kleef M, Patjin J. High prevalence of pain in patients with cancer in a large population-based study in the Netherlands. Pain. 2007:132, 312-320

14. Chen Z, Maricic M, Bassford TL, Pettinger M, Ritenbaugh C, Lopez AM, Barad DH, Gass M, Leboff MS (2005) Fracture risk among breast cancer survivors: Results from the Women's Health Initiative Observational Study. Arch Intern Med 165(5):552-558. PubMed PMID: 15767532

15. Black DM, Geiger EJ, Eastell R, Vittinghof E, Li BH, Rayn DS, Dell RM, Adams AL (2020) Atypical femur fracture risk versus fragility fracture prevention with bisphosphonates. N Engl J Med 383:743753. doi:10.1056/NEJMoa1916525

16. Dell RM, Adams AL, Greene DF et al (2012) Incidence of atypical nontraumatic diaphyseal fractures of the femur. J Bone Miner Res 27:2544-2550

17. Schilcher J, Koeppen V, Aspenberg P, Michaëlsson K (2014) Risk of atypical femoral fracture during and after bisphosphonate use. N Engl J Med 371:974-976

18. Fiorentino L, Rissling M, Liu L, Ancoli-Israel S (2011) The symptom cluster of sleep, fatigue and depressive symptoms in breast cancer patients: Severity of the problem and treatment options. Drug Discov Today Dis Models 8(4):167-173

19. Peate $M$, Saunders $C$, Cohen $P$ et al (2021) Who is managing menopausal symptoms, sexual problems, mood and sleep disturbance after breast cancer and is it working? Findings from a large community-based survey of breast cancer survivors. Breast Cancer Res Treat 187:427-435. https://doi.org/10.1007/s10549-021-06117-7

\section{Tables}

Table 1: Characteristics of breast cancer survivors by incident fracture status

Table 2: Tumor characteristics \& breast cancer treatments by incident fracture status 


\begin{tabular}{|c|c|c|c|c|}
\hline & Fractures & & & \\
\hline & No & Yes & Total & \\
\hline & $(\mathrm{N}=19,308)$ & $(\mathrm{N}=2,038)$ & $(N=21,346)$ & \\
\hline & $\mathrm{n}(\%)$ & $n(\%)$ & $\mathrm{n}(\%)$ & $P$ value \\
\hline Stage at diagnosis & & & & $<0.001$ \\
\hline Stage 0 & $4102(21.3)$ & $344(16.9)$ & $4446(20.8)$ & \\
\hline Stage I & $8230(42.6)$ & $976(47.9)$ & $9206(43.1)$ & \\
\hline Stage II & $5376(27.8)$ & $566(27.8)$ & $5942(27.8)$ & \\
\hline Stage III & $1600(8.3)$ & $152(7.5)$ & $1752(8.2)$ & \\
\hline Surgery & $18674(96.7)$ & $1981(97.2)$ & $20655(96.8)$ & 0.238 \\
\hline Adjuvant chemotherapy & $6795(35.2)$ & $569(27.9)$ & $7364(34.5)$ & $<0.001$ \\
\hline Adjuvant endocrine therapy & $11409(59.1)$ & $1271(62.4)$ & $12680(59.4)$ & 0.004 \\
\hline Adjuvant radiation therapy & $8792(45.5)$ & $888(43.6)$ & $9680(45.4)$ & 0.090 \\
\hline
\end{tabular}

Table 3:Use of sleep aid medications among breast cancer survivors during follow-up 


\begin{tabular}{|ll|}
\hline & Sleep aid medications \\
\hline & $\mathbf{N}=\mathbf{8}, 225$ \\
\hline Sleep medication use* & \\
\hline Doxepin & $119(1.5)$ \\
\hline Eszopiclone & $26(0.3)$ \\
\hline Flurazepam & $12(0.2)$ \\
\hline Lorazepam & $5819(70.8)$ \\
\hline Surorexant & $4(0.05)$ \\
\hline Temazepam & $1615(19.6)$ \\
\hline Trazodone & $2296(27.9)$ \\
\hline Triazolam & $72(0.9)$ \\
\hline Zaleplon & $2(0.02)$ \\
\hline Zolpidem & $1540(18.7)$ \\
\hline Cumulative duration (days) & \\
\hline Median (IQR) & $60.0(15.0,268.0)$ \\
\hline *Exceeds 100\% due to use of multiple types \\
\hline
\end{tabular}

Table 4: Psychiatric medications and mental health status in breast cancer survivors during follow-up by sleep aid medication use 
Sleep aid medication use

\begin{tabular}{lll} 
No & Yes & Total \\
\hline$(\mathrm{N}=13,121)$ & $(\mathrm{N}=8,225)$ & $(\mathrm{N}=21,346)$ \\
\hline $\mathrm{n}(\%)$ & $\mathrm{n}(\%)$ & $\mathrm{n}(\%)$ \\
\hline
\end{tabular}

Mental health conditions after breast cancer

Depression

Anxiety

\section{Psychiatric medication use}

Antidepressants only

Anti-anxiety drugs only ${ }^{a}$

Used both drugs

Neither

Antidepressant type ${ }^{b}$

MOAl only

NDRI only

SARI only

SNRI only

SSRI only (Paroxetine, Fluoxetine, other SSRI)

TCA only

TECA only

Other types

Multiple types

Psychiatric drug use, cumulative duration (days)

Median (IQR)

a.Includes: aripiprazole, asenapine, chlorpromazine, clozapine,haloperidol, loxapine,lurasidone,olanzapine, perphenazine, pimozide, prochlorperazine, quetiapine,thioridazine,thiothixene,trifluoperazine, ziprasidone

${ }^{b} \mathrm{MOAl}=$ Monoamine oxidase inhibitors; $\mathrm{NDRI}=$ norepinephrine-dopamine reuptake inhibitor; SARI=Serotonin antagonist and reuptake inhibitors; SNRI=Serotonin and norepinephrine reuptake inhibitors; SSRI= Selective serotonin reuptake inhibitors; TCA=Tricyclic antidepressants; TECA= Tetracyclic antidepressants

$\begin{array}{lll}2255(17.2) & 3327(40.5) & 5582(26.2) \\ 2801(21.4) & 4712(57.3) & 7513(35.2)\end{array}$

$2625(20.0) \quad 2510(30.5) \quad 5135(24.1)$

$1849(14.1) \quad 1627(19.8) \quad 3476(16.3)$

$827(6.3) \quad 2205(26.8) \quad 3032(14.2)$

$7820(59.6) \quad 1883(22.9) \quad 9703(45.5)$

\begin{tabular}{lll}
\hline $80(0.6)$ & $73(0.9)$ & $153(0.7)$ \\
\hline $1(0.01)$ & $1(0.01)$ & $2(0.01)$ \\
\hline $385(2.9)$ & $246(3.0)$ & $631(3.0)$ \\
\hline $885(6.7)$ & $748(9.1)$ & $1633(7.7)$ \\
\hline $581(4.4)$ & $314(3.8)$ & $895(4.2)$ \\
\hline $61(0.5)$ & $58(0.7)$ & $119(0.6)$ \\
\hline $21(0.2)$ & $24(0.3)$ & $45(0.2)$ \\
\hline $607(4.6)$ & $1046(12.7)$ & $1653(7.7)$
\end{tabular}

$61.0(6.0,569.0)$ 
Table 5: The association of sleep medications use and risk of fractures among breast cancer survivors in based on full cohort ${ }^{a}$ and subset of new users ${ }^{b}$ of sleep aid medications

\section{Supplementary Files}

This is a list of supplementary files associated with this preprint. Click to download.

- SUPPLEMENTALTABLE.docx 